\title{
Identification of two novel mutations in the COMP gene in six families with pseudoachondroplasia
}

\author{
WEI-JIA YU ${ }^{1 *}$, ZENG ZHANG $^{2 *}$, JIN-WEI HE $^{1}$, WEN-ZHEN FU ${ }^{1}$, CHUN WANG $^{1}$ and ZHEN-LIN ZHANG ${ }^{1}$ \\ ${ }^{1}$ Department of Osteoporosis and Bone Diseases, Metabolic Bone Disease and Genetic Research Unit; \\ ${ }^{2}$ Department of Orthopedic Surgery, Sixth People's Hospital Affiliated to Shanghai Jiao Tong University, \\ Shanghai 200233, P.R. China
}

Received May 31, 2015; Accepted April 13, 2016

DOI: $10.3892 / \mathrm{mmr} .2016 .5486$

\begin{abstract}
Pseudoachondroplasia (PSACH; MIM no. 177170) is an autosomal dominant osteochondrodysplasia characterized by short-limb short stature, brachydactyly and early-onset osteoarthropathy. Typically, at approximately two years of age, the rate of growth falls below the standard growth curve, causing a moderately severe form of disproportionate short-limb short stature. The current study described the clinical and radiographic observations of six Chinese patients with PSACH, and identified two de novo novel missense mutations [p.Asp326Asn (c.976G>A) and c.1585A>G (p.Thr529Ala)] in cartilage oligomeric matrix protein (COMP) in the patients. The current study expanded the mutation spectrum of the COMP gene, and contributes to the understanding of phenotype/genotype of COMP-associated diseases.
\end{abstract}

\section{Introduction}

Pseudoachondroplasia (PSACH; MIM no. 177170) is an autosomal dominant osteochondrodysplasia characterized by short-limb short stature, brachydactyly and early-onset osteoarthropathy (1). Affected individuals appear normal at birth. A waddling gait is often the presenting feature at the onset of walking (1). Typically, at approximately two years of age, the rate of growth falls below the standard growth curve, causing a moderately severe form of disproportionate short-limb short stature (1). Vertebral anomalies, present in childhood, usually

Correspondence to: Professor Zhen-Lin Zhang or Professor Chun Wang, Department of Osteoporosis and Bone Diseases, Metabolic Bone Disease and Genetic Research Unit, Sixth People's Hospital Affiliated to Shanghai Jiao Tong University, 600 Yi-Shan Road, Shanghai 200233, P.R China

E-mail: zzl2002@medmail.com.cn

E-mail: chunwang66@gmail.com

\section{${ }^{*}$ Contributed equally}

Key words: cartilage oligomeric matrix protein, $\mathrm{COMP}, \mathrm{PSACH}$, pseudoachondroplasia resolve with age, however osteoarthritis is progressive and severe.

Previous genetic studies have demonstrated that PSACH is caused by heterozygous mutations in the cartilage oligomeric matrix protein (COMP; MIM no. 600310) gene (2,3). COMP is located on chromosome 19p13.1 (4). COMP rat and bovine sequencing indicate that it is a member of the thrombospondin gene family (4). It contains 19 exons, encoding the epidermal growth factor-like (type II) repeats, calmodulin-like (type III) repeats and the C-terminal domain. Exons 4-19 correspond in sequence and intron location to the thrombospondin genes, whereas exons 1-3 are unique to COMP (2).

It is notable that mutations in COMP produce clinical phenotypes ranging from the severe end of the spectrum, $\mathrm{PSACH}$, to a mild condition, multiple epiphyseal dysplasia (MED). Briggs et al (5) suggested that missense mutations in the type III repeats (amino acid residues 268-528) are the major cause of PSACH and MED, exhibit significant phenotypic correlations and derived preliminary genotype to phenotype correlations. However, the correlations remain unclear, and more data is required to further deduce genotype-phenotype correlations. Although numerous mutations in COMP in patients with PSACH have been reported, data on Chinese patients with PSACH remain limited (6-10). The current study presents the molecular and clinical characteristics of six Chinese patients with PSACH.

\section{Materials and methods}

Patients. The current study was approved by the Ethics Committee of the Sixth People's Hospital Affiliated to Shanghai Jiao Tong University (Shanghai, China). Prior to initiation of the study, written informed consent for genetic tests and publication of the case details was obtained from all adult participants and the parents or the legal guardians on behalf of children involved in the study.

Mutation analysis. Informed consent was obtained from the families and from 250 healthy ethnically matched volunteers prior to blood sampling and DNA analysis. These 250 Chinese volunteers (125 males and 125 females) were recruited from the Department of Osteoporosis, Sixth People's Hospital Affiliated to Shanghai Jiao Tong University. This is the same control cohort 
Table I. Polymerase chain reaction primer sequences of the COMP gene.

\begin{tabular}{lll}
\hline Gene & \multicolumn{1}{c}{ Forward primer } & Reverse primer \\
\hline COMP-1 & 5'-GACGCTGGTGACTCTGTTTCC-3' & 5'-GGGCCTATTTATCCCCAAGG-3' \\
COMP-2 & 5'-GCAAGAGGATGCGATACAGCCACACC-3' & 5'-AGCCTGGATCCCGGGCCTCTC-3' \\
COMP-3 & 5'-GGAGGTCCGGGTTCGCTGCAA-3' & 5'-CCATTCCCGTTCGTCCGTCCA-3' \\
COMP-4 & 5'-ACGGGGCAGAAAAGCGGGACA-3' & 5'-CGGGGATGGTCCTTGGGTTGG-3' \\
COMP-5 & 5'-TGCCCGCCCTCATCCTTCCTC-3' & 5'-TCACCACCCCACGCAGACACC-3' \\
COMP-6 & 5'-CCCTCCTCCCCCAGTGCAACG-3' & 5'-ACGCCTGGTCGCCCACGAAG-3' \\
COMP-7 & 5'-GGTAAGGCCCGCTGGGGAGGA-3' & 5'-GGGCGGGCACAGAAGGTGTGA-3' \\
COMP-8 & 5'-GGAGCGCCAGTGCCGTAAGGTG-3' & 5'-TCCCTAGGCCCCGCTCACAGC-3' \\
COMP-9 & 5'-GATGGGGCGTGGCTTGGATGA-3' & 5'-CTGGCGCCCCACCATGGTCTT-3' \\
COMP-10 & 5'-TGAAGTTGGGACTCTGTTCCAG-3' & 5'-TGGATAGGTGGGATCCAGAGA-3' \\
COMP-11 & 5'-AGCCTGCGGTGGGGGTTTCCT-3' & 5'-CCACGCCGTCCCCTGAGAGGT-3' \\
COMP-12 & 5'-CCACGATGGCCAGGGTGATGC-3' & 5'-CCCGCTCCGTGGCAGGATAGC-3' \\
COMP-13 & 5'-GGGCGGGCCCTGACTTTAGCC-3' & 5'-CCCTCTGTCCCCGCCCTTCCT-3' \\
COMP-14 & 5'-GGAGGGGCGTGGCCAATGGT-3' & 5'-GCCCAGAGGAGGGCTGGGACA-3' \\
COMP-15 & 5'-CTGTGACCTATCCCCAAGCTG-3' & 5'-GGCTCATTCTAACTGCCCTGT-3' \\
COMP-16 & 5'-CCAGTCAGGGGTACCCATCTT-3' & 5'-AGCACTGAGGCCTTGGTTTG-3'
\end{tabular}

COMP, cartilage oligomeric matrix protein.

used in our previous studies $(11,12)$. The DNA was extracted from peripheral white blood cells by proteinase $\mathrm{K}$ (Kurabo Industries, Ltd., Tokyo, Japan) digestion followed by purification with phenol/chloroform and isopropyl alcohol precipitation (Ling Feng Chemical Reagent Co., Ltd., Shanghai, China). The DNA sequence for the COMP gene was obtained from the available online database (GenBank accession no. NC_000019). The COMP gene primers (Table I) were designed using Primer3 software (version 0.4.0; http://primer3. wi.mit.edu/). Template DNA extracted from peripheral white blood cells, and all exons and their exon-intron boundaries in the COMP gene were amplified via polymerase chain reaction (PCR). The reaction mixture for COMP 10F/R was as follows: $20 \mu \mathrm{l}$ Mixture including 1X GC buffer I (Takara Bio, Inc., Otsu, Japan), $2.5 \mathrm{mM} \mathrm{Mg}{ }^{2+}, 0.2 \mathrm{mM}$ dNTP, $0.2 \mu \mathrm{M}$ of each primer, 1 unit HotStarTaq polymerase (Qiagen, Inc., Valencia, CA, USA) and $1 \mu \mathrm{l}$ template DNA. The reaction mixture for COMP 1F/R, 15F/R and 16F/R was as follows: A $20 \mu 1$ mixture including 1X HotStarTaq buffer, $2.0 \mathrm{mM} \mathrm{Mg}^{2+}, 0.2 \mathrm{mM} \mathrm{dNTP}$, $0.2 \mu \mathrm{M}$ of each primer, 1 unit HotStarTaq polymerase and $1 \mu \mathrm{l}$ template DNA. The reaction mixture for the remaining fragments was as follows: A $20 \mu 1$ mixture including $1 \mathrm{X}$ GC buffer I (Takara Bio, Inc.), $2.5 \mathrm{mM} \mathrm{Mg}{ }^{2+}, 0.2 \mathrm{mM}$ dNTP, $0.2 \mu \mathrm{M}$ each primer, 1 unit HotStarTaq polymerase and $1 \mu 1$ template DNA. The thermal cycling conditions for COMP $10 \mathrm{~F} / \mathrm{R}$ were as follows: $95^{\circ} \mathrm{C}$ for $15 \mathrm{~min}$; 11 cycles of $94^{\circ} \mathrm{C}$ for $15 \mathrm{sec}, 62-0.5^{\circ} \mathrm{C}$ per cycle for $40 \mathrm{sec}, 72^{\circ} \mathrm{C}$ for $1 \mathrm{~min} ; 24$ cycles of $94^{\circ} \mathrm{C}$ for $15 \mathrm{sec}, 56^{\circ} \mathrm{C}$ for $30 \mathrm{sec}, 72^{\circ} \mathrm{C}$ for $1 \mathrm{~min}$; and $72^{\circ} \mathrm{C}$ for $2 \mathrm{~min}$. The thermal cycling conditions for COMP $1 \mathrm{~F} / \mathrm{R}, 15 \mathrm{~F} / \mathrm{R}$ and $16 \mathrm{~F} / \mathrm{R}$ were as follows: $95^{\circ} \mathrm{C}$ for $15 \mathrm{~min}$; 11 cycles of $94^{\circ} \mathrm{C}$ for $15 \mathrm{sec}$, $62-0.5^{\circ} \mathrm{C}$ per cycle for $40 \mathrm{sec}, 72^{\circ} \mathrm{C}$ for $1 \mathrm{~min} ; 24$ cycles of $94^{\circ} \mathrm{C}$ for $15 \mathrm{sec}, 56^{\circ} \mathrm{C}$ for $30 \mathrm{sec}, 72^{\circ} \mathrm{C}$ for $1 \mathrm{~min}$; and $72^{\circ} \mathrm{C}$ for $2 \mathrm{~min}$. The thermal cycling conditions for the remaining fragments were as follows: $95^{\circ} \mathrm{C}$ for $15 \mathrm{~min} ; 35$ cycles of $96^{\circ} \mathrm{C}$ for $10 \mathrm{sec}$ and $68^{\circ} \mathrm{C}$ for $4 \mathrm{~min}$. Direct sequencing was performed using the BigDye Terminator Cycle Sequencing Ready Reaction kit, version 3.1 (Applied Biosystems; Thermo Fisher Scientific, Inc., Waltham, MA, USA), and the sequencing was analyzed with an ABI Prism 3130 automated sequencer (Applier Biosystems; Thermo Fisher Scientific, Inc.). To assess the damaging effects of missense mutations in silico, the online database, Protein Variation Effect Analyzer (PROVEAN; http://provean.jcvi. org/index.php) was used (13).

\section{Results}

Patients. The present study included six Chinese families with PSACH. Detailed clinical information of these families is described in the following sections, and the clinical characteristics are summarized in Table II.

Family 1. The proband was a 20-year-old male of Han ethnicity. He was the second of two siblings in a healthy non-consanguineous couple. The pregnancy and delivery of this individual were considered normal, and no abnormalities were identified at birth. The patient began to walk at the age of 16 months, and slowness in linear growth was noticed at 5 years of age. Simultaneously, a limp and brachydactyly were noted. Physical examination at the age of 20 years demonstrated disproportional short stature with short limbs: Height, $149 \mathrm{~cm}$ (-3 standard deviations); arm span, $137 \mathrm{~cm}$. Generalized brachydactyly of the hands was observed. No restricted extension of the joints was identified, and intelligence was considered normal. The radiographic images presented in Fig. 1A exhibited clinical features of PSACH disease. The patient exhibited vertebral bodies of a normal height (anteroposterior and lateral views), flat bilateral femoral capitals of the pelvis (anteroposterior view), mild osteoarthritis of the left knee (anteroposterior and lateral views) and a hand with brachydactyly (posterior-anterior 


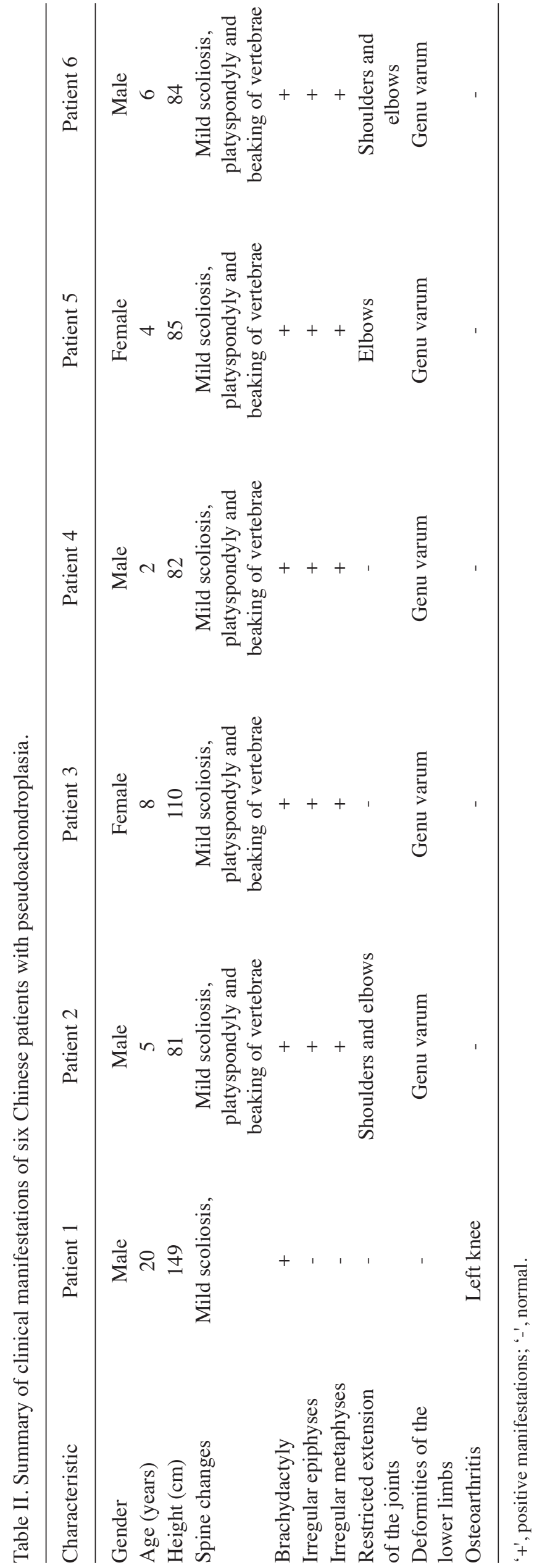

view). His parents, brother and relatives were healthy with no similar features observed.

Family 2. The proband was a 5-year-old Han boy. He was the only child of a healthy non-consanguineous couple. The pregnancy and delivery of this individual were considered normal, however no birth parameters were available. Genu varum and slowness in linear growth were noted at the age of 20 months, and the individual began to walk at the age of 2. Physical examination at age of 5 observed disproportional short stature with short limbs: Height, $81 \mathrm{~cm} \mathrm{(-3} \mathrm{standard} \mathrm{devia-}$ tions); arm span, $78 \mathrm{~cm}$; upper segment/lower segment ratio of 1.57 . The hands exhibited generalized brachydactyly and the shoulders and elbows presented with restricted extension. clear genu varum was noted and intelligence was considered normal. The radiographic images presented in Fig. 1B exhibited clinical features of PSACH disease. The patient presented with mild scoliosis, platyspondyly and anterior beaking of the vertebral bodies (anteroposterior and lateral views), small capital femoral epiphyses of the pelvis (anteroposterior view), irregular epiphyses in the knees (anteroposterior view) and a hand with brachydactyly (posterior-anterior view). All of his parents and relatives were healthy with no similar features.

Family 3. The 8-year-old proband was born to non-consanguineous and healthy parents. She was born at full term with a birth height of $51 \mathrm{~cm}$. A waddling gait was recognized upon learning to walk, and during physical examination at 8 years of age, her height was measured as $110 \mathrm{~cm}$ (-3 standard deviations). Genu varum was clear and moderate generalized brachydactyly of the hands was observed. The extension of the shoulders and elbows was restricted and the individual additionally presented with pectus carinatum. Her intelligence was normal. The radiographic images presented in Fig. 1C exhibited clinical features of PSACH disease. The patient had similar radiographic characteristics to that of patient 2. Laboratory tests including mucopolysaccharide analysis of urine and thyroid function tests were normal, and all of her parents and relatives were healthy with no similar features.

Family 4. The proband was a 2-year-old (35-month-old) Han boy. He was the first of two siblings in a healthy non-consanguineous couple. Results of a prenatal fetal ultrasound were normal, as was the delivery of this child. His birth weight was $3.2 \mathrm{~kg}$ and his birth height was $52 \mathrm{~cm}$. He manifested with a delayed odontogenesis at 6 months. The anterior fontanelle closed at 17 months. He was unable to walk until 19 months of age. Physical examination observed disproportional short stature with short limbs: Height, $82 \mathrm{~cm}$ (-3 standard deviations); arm span, $78 \mathrm{~cm}$; upper segment/lower segment ratio, 1.73 . He additionally presented with clear pectus carinatum and genu varum. His enunciation was neither clear nor continuous, however he responded well. The radiographic images presented in Fig. 1D exhibited clinical features of PSACH disease. The patient had similar radiographic characteristics to that of patient 2 , however irregular metaphyses of the left elbow (anteroposterior view) was additionally observed. His parents and relatives were healthy with no similar features observed. 


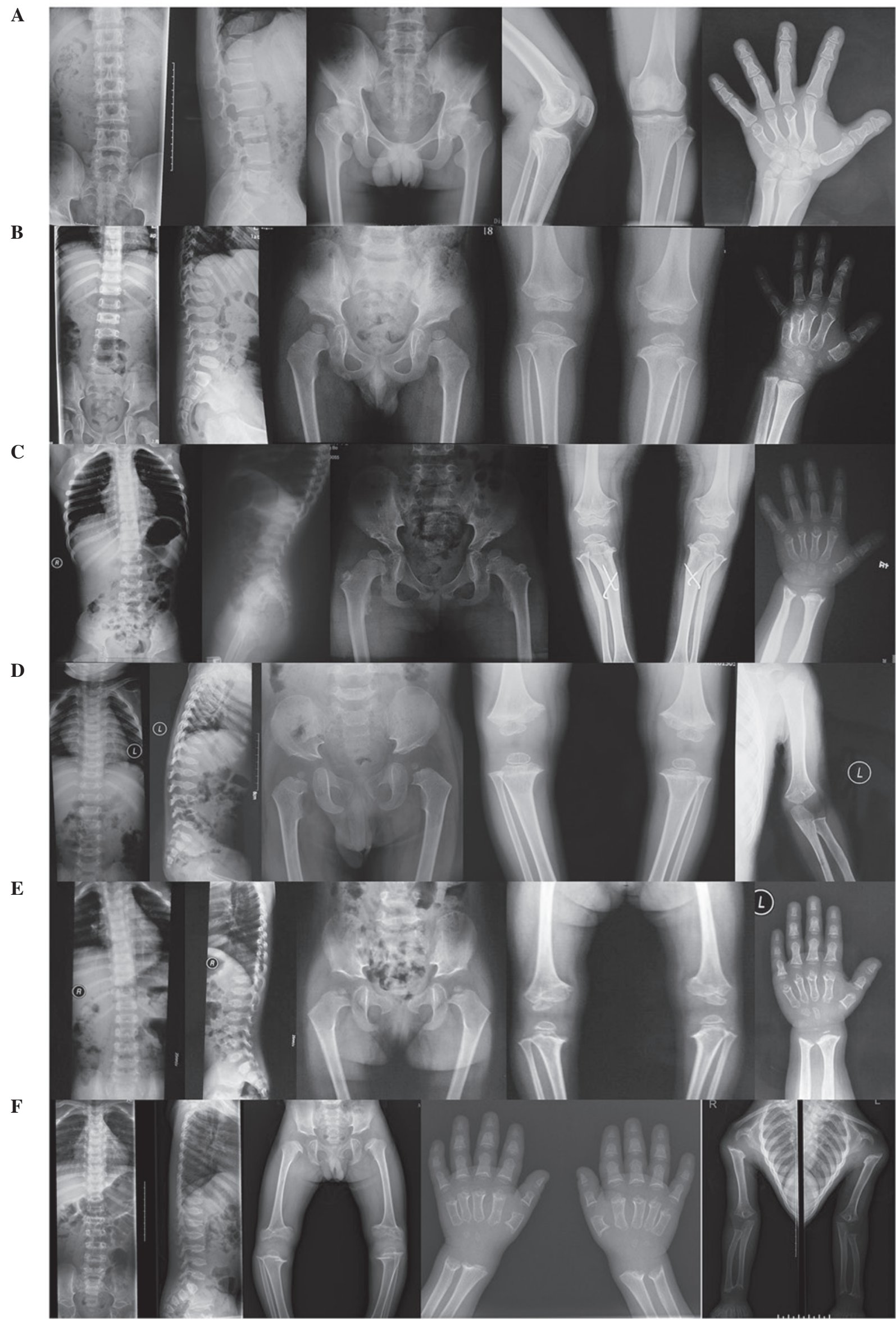

Figure 1. Radiographic images from the patients. (A-F) represent patients 1-6, respectively. In all patients, brachydactyly was observed. (A) Mild scoliosis of the spine, osteoarthritis of the left knee and normal height of vertebral bodies. (B) Mild scoliosis, platyspondyly and anterior beaking of the vertebral bodies, irregular epiphyses and metaphyses and small capital femoral epiphyses. (C-F) Exhibit similar radiographic characteristics to that of (B). 
Family 5. The proband was a 4-year-old Han girl. She was the second of three siblings in a healthy nonconsanguineous couple. The pregnancy and delivery of this individual were considered normal. She was born with a birth weight of $5 \mathrm{~kg}$ and a birth height of $50 \mathrm{~cm}$. Developmental progress was normal until 1.5 years of age, when the patient learnt to walk and presented with mild bowing of the legs. Slowness in linear growth was noted at 2.5 years, with the individual reported to be of short stature in comparison with her peers. Physical examination at the age of 4 years demonstrated disproportional short stature with short limbs: Height, $85 \mathrm{~cm}$ (-3 standard deviations); arm span, $72 \mathrm{~cm}$; upper segment/lower segment ratio, 1.43 . There was moderate generalized brachydactyly of the hands and restricted extension of the elbows and genu varum were clear. Her intelligence was normal. The radiographic images presented in Fig. 1E exhibited clinical features of PSACH disease. The patient had similar radiographic characteristics to that of patient 2 . Her parents and relatives were healthy with no similar features.

Family 6. The proband was a 6-year-old Han boy. He was the only child of a healthy non-consanguineous couple. The pregnancy and delivery of this individual were considered normal, however no birth parameters were available Genu varum and slowness in linear growth were noted at the age of 2. Physical examination demonstrated disproportional short stature with short limbs: Height, $84 \mathrm{~cm}(-3$ standard deviations); arm span, $83 \mathrm{~cm}$; upper segment/lower segment ratio, 1.51 . Moderate generalized brachydactyly of the hand was observed and extension of the shoulders and elbows was restricted. He additionally presented with pectus carinatum. His intelligence was normal. The radiographic images presented in Fig. 1F exhibited clinical features of PSACH disease. The patient had similar radiographic characteristics to that of patient 2. His parents and relatives were healthy with no similar features.

Sequence analysis. Screening was conducted for mutations in COMP in all patients and their parents using PCR followed by direct sequence analysis. The sequencing identified that five patients carried heterozygous missense mutations of COMP and one carried a heterozygous in-frame deletion in exon 13 of COMP: c.772G $>\mathrm{C}$ (p.Gly258Arg) in exon 8 of patient 1 (Fig. 2A), c.1585A $>$ G (p.Thr529Ala) in exon 14 of patient 2 (Fig. 2B), c.976G $>$ A (p.Asp326Asn) in exon 10 of patient 3 (Fig. 2C), c.1417_1419delGAC (p.Asp473del) in exon 13 of patient 4 (Fig. 2D), c.812A $>$ T (p.Asp271Val) in exon 8 of patient 5 (Fig. 2E), and c.1512C >G (p.Cys504Trp) in exon 14 of patient 6 (Fig. 2F). Based on DNA sequence analysis, all the mutations were de novo, and were not identified in DNA samples from healthy parents and 250 healthy volunteers. Among these mutations, c.976G $>$ A (p.Asp326Asn) and c.1585A $>$ G (p.Thr529Ala) are, to the best of our knowledge, novel. p.Asp326Asn and p.Thr529Ala were non-conservative, evolutionarily highly conserved amino acids from fish to mammals (Fig. 3A and B), and were predicted in silico by PROVEAN to be of pathogenic relevance (p.Asp326Asn, PROVEAN score of -4.542 ; p.Thr529Ala, PROVEAN score of -4.610).

\section{Discussion}

Although PSACH is an unambiguous skeletal dysplasia easily diagnosed by physicians, two major entities should be considered in the differential diagnosis. Firstly, the body proportions of patients with PSACH resemble those of patients with achondroplasia at the second year of life or later. However, patients with PSACH appear normal at birth and their faces are normal, while the characteristics of achondroplasia are recognized at birth with characteristic frontal bossing (14). In addition, there is gradual narrowing of the lumbar interpedicular distances in the lower lumbar spine in achondroplasia, which is absent in PSACH. Secondly, mucopolysaccharidosis IV, also termed Morquio syndrome, may be confused with PSACH, due to the fact that both disorders present with anterior beaking of the vertebral bodies (15). However, the anterior beaking of the vertebral bodies is seen exclusively during childhood in patients with PSACH, and resolves with age. In addition, pointed proximal $2 \mathrm{nd}$, 3rd, 4th and 5th metacarpals, which are unique to mucopolysaccharidosis IV, are not observed in PSACH. Mucopolysaccharide analysis of urine will confirm diagnosis. Mutation analysis is, however, a simple way of confirming the diagnosis of PSACH.

Previous ultrastructural studies on cartilage from patients with PSACH demonstrated abnormal accumulation of electron-dense and light material within the chondrocyte rough endoplasmic reticulum $(16,17)$. Biochemical and histochemical studies have additionally suggested that PSACH was a generalized cartilage disorder involving abnormalities of proteoglycans $(18,19)$. Cartilage consists of chondrocytes and abundant extracellular matrix components including glycoproteins, aggrecans and collagens. COMP is a pentameric glycoprotein of the cartilage extracellular matrix that is localized predominantly to the territorial matrix surrounding the chondrocytes. In vitro studies have reported that chondrocyte-specific intracellular trafficking defects were observed for the p.Asp469del mutant COMP $(20,21)$. Furthermore, different COMP mutations in the type 3 repeat domain have been identified to produce variable effects on intracellular transport, which correlate with clinical severity (22). Mutations in COMP have additionally been reported to affect the secretion of type IX collagen and matrilin 3, however not the secretion of aggrecan and type II collagen (23). Furthermore, a recent study using the COMP p.Asp469del mutant mouse model suggested that chondrocyte stress triggered by the expression of mutant COMP was central to the pathogenesis of PSACH (24).

The key observation of the present study was the identification of two novel mutations in COMP: c. $976 \mathrm{G}>\mathrm{A}$ (p.Asp326Asn) and c.1585A>G (p.Thr529Ala). Notably, previous studies reported that p.Asp326Tyr resulted in PSACH while p.Asp326Gly led to MED $(25,26)$. In the current study, p.Asp326Asn in COMP was observed to result in a PSACH phenotype in a Chinese girl. This patient manifested with severe epiphyseal deformities and clear delay in linear growth. Furthermore, the radiographic images indicated clear platyspondyly and anterior beaking of the vertebral bodies. These features can help differentiate between PSACH from MED. It is suggested that different substitutions at the same residue of p.Asp326 lead to 
A

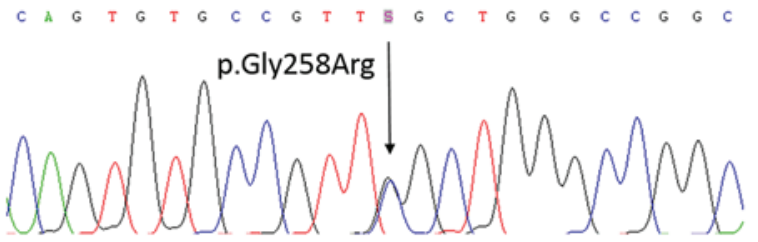

B

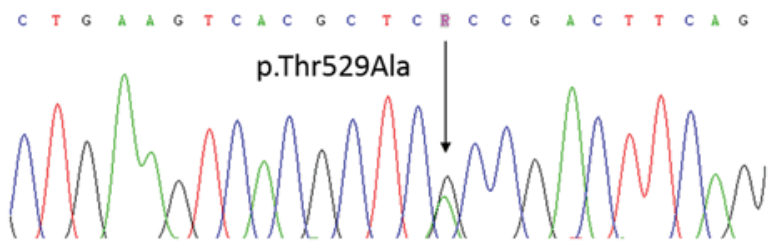

D

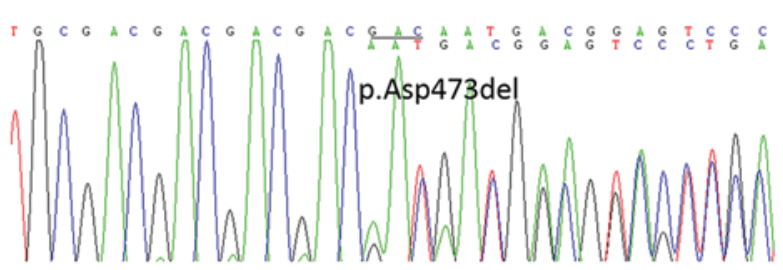

F

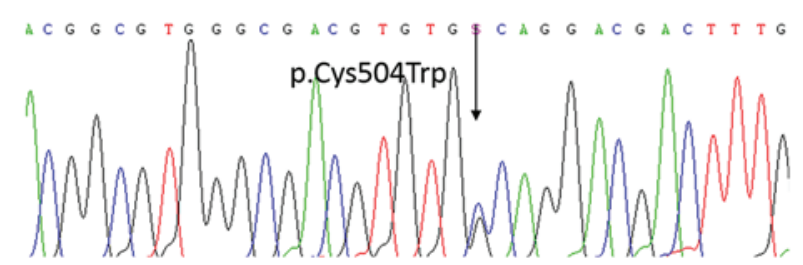

Figure 2. Genetic analyses of the COMP gene in the six patients. (A) c.772G $>C$ (p.Gly258Arg) in exon 8 of patient 1. (B) c.1585A $>$ G (p.Thr529Ala) in exon 14 of patient 2. (C) c.976G $>$ A (p.Asp326Asn) in exon 10 of patient 3. (D) c.1417_1419delGAC (p.Asp473del) in exon 13 of patient 4. (E) c.812A $>$ T (p.Asp271Val) in exon 8 of patient 5. (F) c.1512C >G (p.Cys504Trp) in exon 14 of patient 6. Nucleotide and amino acid positions are written according to Refseq NM_000095.2 and NP_000086.2. COMP, cartilage oligomeric matrix protein.

A

Homo sapiens Mus musculus Bos taurus Rattus norvegicus Macaca mulatta Canis familiaris Cavia porcellus Gallus gallus Danio rerio

B

Homo sapiens Mus musculus Bos taurus Rattus norvegicus Macaca mulatta Canis familiaris Cavia porcellus Gallus gallus Danio rerio
p.Asp326

VDRDGIGDACDPDADGDGVPNEKDNCPLVRNPDQRNTDEDKWGDA VDRDGIGDACDPDADGDGVPNEQDNCPLVRNPDQRNSDSDKWGDA VDQDGIGDACDPDADGDGVLNEKDNCPLVRNPDQRNTDGDKWGDA VDRDRIGDACDPDADGDGVPNEQDNCPLVRNPDQRNSDKDKWGDA VDRDGIGDACDPDADGDGVPNEKDNCPLVRNPDQRNTDEDKWGDA VDRDGIGDACDPDADGDGVLNEQDNCKLVRNPDQRNADGDKWGDA VDRDGIGDACDPDADGDGVPNEEDNCPLVRNPDQRNTDNDKWGDA ADRDGIGDACDDDADGDGILNAEDNCVYTRNADQRNADKDNFGDA ADKDNIGDACDDDADGDGILNIEDNCVLVPNVNQKNTDQDDYGDA

$$
\text { p. Thr } 529
$$

DDFDADKVVDKIDVCPENAEVTLTDFRAFQTVVLDPEGDAQIDPN GDFDADKVIDKIDVCPENAEVTLTDFRAFQTVVLDPEGDAQIDPN GDFDADKVVDKIDVCPENAEVTLTDFRAFQTVVLDPEGDAQIDPN GDFDADKVIDKIDVCPENAEVTLTDFRAFQTVVLDPEGDAQIDPN GDFDADKVVDKIDVCPENAEVTLTDFRAFQTVVLDPEGDAQIDPN GDFDADKVVDKIDVCPENAEVTLTDFRAFQTVVLDPEGDAQIDPN GDFDADKVVDQIDVCPENAEVTLTDFRAFQTVVLDPEGDAQIDPN DDFDRDMVIDRIDVCPENAEVTLTDFRAFQTVVLDPEGDAQIDPN NDFDNDTFSDIIDVCPENAEVTLTDFRTYQTVVLDPEGDAQIDPN

Figure 3. (A) p.Asp326 is evolutionarily highly conserved from fish to mammal. (B) p.Thr529 is evolutionarily highly conserved from fish to mammal.

different phenotypes. It is also possible that genetic modifiers of phenotypic severity are likely to influence disease severity (5). This data may be useful for further analysis of correlations between genotype and phenotype. In addition, a notable hotspot, c.1417_1419delGAC (p.Asp473del), was identified. This mutation had been previously reported in 49 cases of PSACH and was identified as the most common mutation (1). 
In summary, the current study reported the molecular and clinical observations of six Chinese patients with PSACH. Two novel mutations in the COMP gene were identified. The present study expanded upon the mutation spectrum in the COMP gene, and contributed to the understanding of the phenotype/genotype of COMP-associated diseases.

\section{Acknowledgements}

The current study was supported by the National Natural Science Foundation of China (grant nos. 81370978 and 81170803 to Professor Zhen-Lin Zhang and grant no. 81200646), the National Basic Research Program of China (grant no. 2014CB942903), Shanghai Leading Talents Award (grant no. 051 to Professor Zhen-Lin Zhang), the Science and Technology Commission of Shanghai Municipality (grant no. 14JC140500 to Professor Zhen-Lin Zhang), Shanghai Municipal Commission of Health and Family Planning (grant no. 2014ZYJB0009), and the Science and Technology Commission of Chongqing Municipality (grant no. CSTC2013jcyjC00009 to Professor Zhen-Lin Zhang).

\section{References}

1. Briggs MD and Chapman KL: Pseudoachondroplasia and multiple epiphyseal dysplasia: Mutation review, molecular interactions, and genotype to phenotype correlations. Hum Mutat 19 465-478, 2002.

2. Briggs MD, Hoffman SM, King LM, Olsen AS, Mohrenweiser H, Leroy JG, Mortier GR, Rimoin DL, Lachman RS, Gaines ES et al: Pseudoachondroplasia and multiple epiphyseal dysplasia due to mutations in the cartilage oligomeric matrix protein gene. Nat Genet 10: 330-336, 1995.

3. Hecht JT, Nelson LD, Crowder E, Wang Y, Elder FF, Harrison WR, Francomano CA, Prange CK, Lennon GG, Deere M, et al: Mutations in exon 17B of cartilage oligomeric matrix protein (COMP) cause pseudoachondroplasia. Nat Genet 10: 325-329, 1995.

4. Newton G, Weremowicz S, Morton CC, Copeland NG, Gilbert DJ, Jenkins NA and Lawler J: Characterization of human and mouse cartilage oligomeric matrix protein. Genomics 24: 435-439, 1994.

5. Briggs MD, Brock J, Ramsden SC and Bell PA: Genotype to phenotype correlations in cartilage oligomeric matrix protein associated chondrodysplasias. Eur J Hum Genet 22: 1278-1282, 2014.

6. Xie X, Liao L, Gao J and Luo X: A novel COMP mutation in a Chinese patient with pseudoachondroplasia. Gene 522: 102-106, 2013.

7. Dai L, Xie L, Wang Y, Mao M, Li N, Zhu J, Kim C and Zhang Y: A novel COMP mutation in a pseudoachondroplasia family of Chinese origin. BMC Med Genet 12: 72, 2011.

8. Cao LH, Wang LB, Wang SS, Ma HW, Ji CY and Luo Y: Identification of novel and recurrent mutations in the calcium binding type III repeats of cartilage oligomeric matrix protein in patients with pseudoachondroplasia. Genet Mol Res 10: 955-963, 2011.
9. Lu CT, Guo L, Zahng ZH, Lin WX, Song YZ and Feng L: Clinical features and COMP gene mutation in a family with a pseudoachondroplasia child. Zhongguo Dang Dai Er Ke Za Zhi 15: 937-941, 2013 (In Chinese).

10. Liu FX, Li ZL, Wei ZJ, Meng Y, Ren CA, Zhang XD, Yu MX and Huang SZ: Genetic analysis and serum level of cartilage oligomeric matrix protein in patients with pseudoachondroplasia. Chin Med J (Engl) 123: 2181-2184, 2010.

11. Yue H, Yu JB, He JW, Zhang Z, Fu WZ, Zhang H, Wang C, $\mathrm{Hu}$ WW, Gu JM, Hu YQ, et al: Identification of two novel mutations in the PHEX gene in Chinese patients with hypophosphatemic rickets/osteomalacia. PLoS One 9: e97830, 2014.

12. Zhang Z, He JW, Fu WZ, Zhang CQ and Zhang ZL: Mutations in the SLCO2A1 gene and primary hypertrophic osteoarthropathy: A clinical and biochemical characterization. J Clin Endocrinol Metab 98: E923-E933, 2013.

13. Choi Y, Sims GE, Murphy S, Miller JR and Chan AP: Predicting the functional effect of amino acid substitutions and indels. PLoS One 7: e46688, 2012.

14. Hunter AG, Hecht JT and Scott CI Jr: Standard weight for height curves in achondroplasia. Am J Med Genet 62: 255-261, 1996.

15. Northover H, Cowie RA and Wraith JE: Mucopolysaccharidosis type IVA (Morquio syndrome): A clinical review. J Inherit Metab Dis 19: 357-365, 1996.

16. Maynard JA, Cooper RR and Ponseti IV: A unique rough surfaced endoplasmic reticulum inclusion in pseudoachondroplasia. Lab Invest 26: 40-44, 1972.

17. Merritt TM, Bick R, Poindexter BJ, Alcorn JL and Hecht JT: Unique matrix structure in the rough endoplasmic reticulum cisternae of pseudoachondroplasia chondrocytes. Am J Pathol 170: 293-300, 2007.

18. Pedrini-Mille A, Maynard JA and Pedrini VA: Pseudoachondroplasia: Biochemical and histochemical studies of cartilage. J Bone Joint Surg Am 66: 1408-1414, 1984.

19. Stanescu V, Maroteaux P, Stanescu R: The biochemical defect of pseudoachondroplasia. Eur J Pediatr 138: 221-255, 1982.

20. Dinser R, Zaucke F, Kreppel F, Hultenby K, Kochanek S, Paulsson M and Maurer P: Pseudoachondroplasia is caused through both intra- and extracellular pathogenic pathways. J Clin Invest 110: 505-513, 2002.

21. Chen TL, Stevens JW, Cole WG, Hecht JT and Vertel BM: Cell-type specific trafficking of expressed mutant COMP in a cell culture model for PSACH. Matrix Biol 23: 433-444, 2004.

22. Chen TL, Posey KL, Hecht JT and Vertel BM: COMP mutations: Domain-dependent relationship between abnormal chondrocyte trafficking and clinical PSACH and MED phenotypes. J Cell Biochem 103: 778-787, 2008.

23. Hecht JT, Hayes E, Haynes R and Cole WG: COMP mutations, chondrocyte function and cartilage matrix. Matrix Biol 23: $525-533,2005$.

24. Suleman F, Gualeni B, Gregson HJ, Leighton MP, Piróg KA, Edwards S, Holden P, Boot-Handford RP and Briggs MD: A novel form of chondrocyte stress is triggered by a COMP mutation causing pseudoachondroplasia. Hum Mutat 33: 218-231, 2012.

25. Jackson GC, Mittaz-Crettol L, Taylor JA, Mortier GR, Spranger J, Zabel B, Le Merrer M, Cormier-Daire V, Hall CM, Offiah A, et al: Pseudoachondroplasia and multiple epiphyseal dysplasia: A 7-year comprehensive analysis of the known disease genes identify novel and recurrent mutations and provides an accurate assessment of their relative contribution. Hum Mutat 33: 144-157, 2012.

26. Zankl A, Jackson GC, Crettol LM, Taylor J, Elles R, Mortier GR, Spranger J, Zabel B, Unger S, Merrer ML, et al: Preselection of cases through expert clinical and radiological review significantly increases mutation detection rate in multiple epiphyseal dysplasia. Eur J Hum Genet 15: 150-154, 2007. 